\title{
OPTIMAL RELAYS COORDINATION EFFICIENT METHOD IN INTERCONNECTED POWER SYSTEMS
}

\author{
Reza Mohammadi * - Hossein Askarian Abyaneh* \\ Farzad Razavi $^{* *}$ - Majid Al-Dabbagh ${ }^{* * *}$ - Seyed H. H. Sadeghi ${ }^{*}$
}

\begin{abstract}
In this paper an efficient method for optimal overcurrent relay coordination is proposed. In the proposed method using genetic algorithm, an objective function (OF) is developed to solve the problem of miscoordination for six pairs short circuit fault currents. The new OF is improved to be simple, optimal and efficient. This technique takes into account the changes in network configuration. The efficiency of the proposed technique is demonstrated by comparing the results of two different networks namely typical 8-bus \& IEEE 30-bus systems with those obtained using the recently developed techniques.
\end{abstract}

K e y w ords: coordination, genetic algorithm, overcurrent relays, protection, relay settings

\section{INTRODUCTION}

Directional overcurrent relays are commonly used as an economical means for protecting power distribution and sub transmission power systems. They are also used as backup protection in transmission systems. The selection of appropriate settings of these relays under various systems conditions plays an important role in timely disconnection of the faulty section of power systems [1].

Overcurrent (OC) relays normally have current setting multipliers ranging from 50 to $200 \%$ in steps of $25 \%$ which is referred to as plug setting (PS). PS for each relay is determined by two parameters: the minimum fault current and the maximum load current [2].

Many attempts have been made in the past to coordinate different types of OC relays with inverse time-current characteristic. The off-line approaches paved the way for the interactive methods. The absence of a systematic procedure and efficient ordering of relays for coordination in all these methods resulted in repeated iterations through all the system relays. To tackle this problem, a heuristic scheme was proposed wherein relays were arranged in a sequence before they were considered for coordination. One more approach proceeded along this line of thought to obtain optimum starting points and an optimum relay sequence using the graph theory concepts. After that have also proposed actual coordination algorithms using the relative sequence matrix (RSM) and set of selection pairs (SSP) for the given transmission network has been developed. Research work on coordination of relays for specific area like radial distribution lines was also reported. Algorithms and methodologies for system-wide coordination were described. Need for subsystem coordination may arise when it is felt to modify the settings of a part of the large system for proper coordination, due to some changes in the system. One such algorithm for subsystem coordination was presented in 1990 [3].

Regarding fault currents pairs, six primary and backup $(\mathrm{P} / \mathrm{B})$ relays current (six pairs) which include different fault locations and different effective line outages have been taken into account using ordinary coordination method [4]. Six current pairs are obtained by putting faults on the places just close to the circuit breaker (CB) of the nearby bus and far end bus with and without lines outages plus high set instantaneous elements consideration. The summarized description of six current pairs is given in section 4 after equation 4 .

Optimal coordination algorithms consider different techniques, both for interconnected and radial industrial networks [3]. Researchers have described various optimization methods to find the directional OC relay settings [3].

Due to the complexity of the nonlinear optimal programming techniques, the traditional optimal coordination of overcurrent relays are commonly performed by linear programming (LP) techniques, including the simplex, two-phase simplex and dual simplex methods [3]. In these methods, the discrimination times $(\Delta)$ of $\mathrm{P} / \mathrm{B}$ relays are considered as constraints. By both constraints and $\mathrm{OF}$ the solution is made.

In reference [1] optimal solution is made by constraints only as well as the comparison between the solution LP and traditional methods results. Minimization is inherently included by setting the time dials to minimum and then increasing their values gradually. It has been revealed that the new method is better than LP and LP method is better than the traditional ordinary method. In reference [5] which has compared LP method with obtained existing traditional method currently used in practice, it has also been resulted that LP method is better

\footnotetext{
* Center of Excelence on Power System, Amirkabir University of Technology, Iran, askarian@aut.ac.ir, ${ }^{* *}$ Tafresh University *** Hydro Tasmania Consulting, Australia
} 


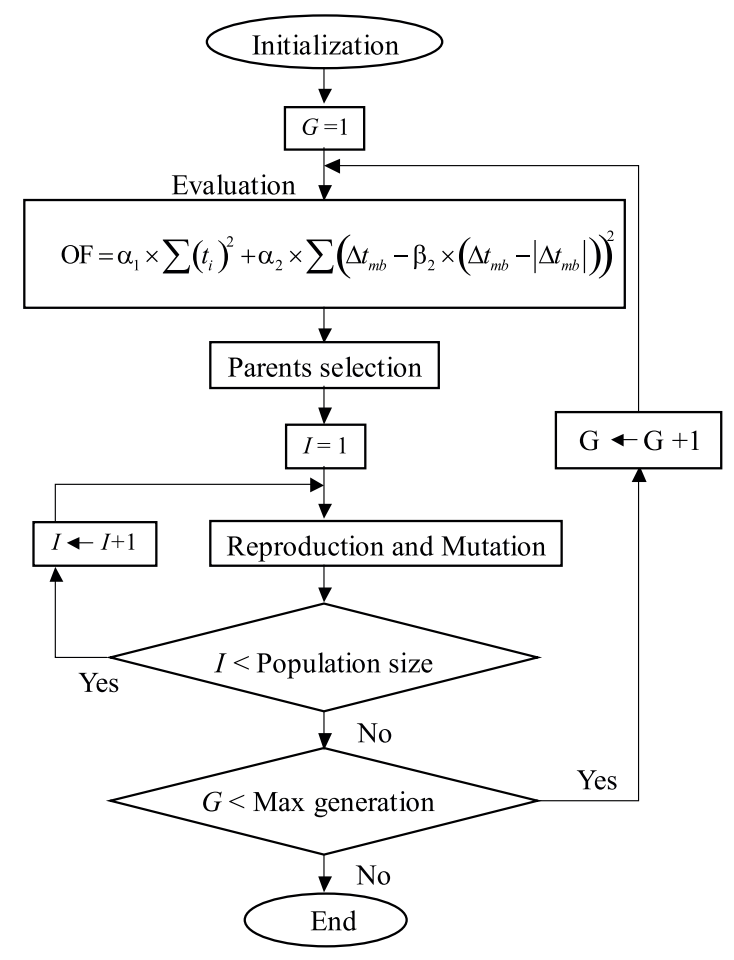

Fig. 1. Flow Diagram of GA Application to Relay Coordination

\begin{tabular}{|l|l|l|l|l|}
\hline Rn TSMn & $\cdots$ & R3 TSM3 & R2 TSM2 & R1 TSM1 \\
\hline
\end{tabular}

Fig. 2. Structure of chromosome

than the existing traditional method. Therefore from references [1] and [5] the superiority of LP method compare to traditional methods currently used has been proven.

In reference [2], a method to recognize the constraints related to six current pairs that make the coordination problem infeasible and then decrease the complexity of the problem of $\mathrm{O} / \mathrm{C}$ relay is given.

Intelligent optimization techniques such as GA can adjust the settings of relays without mentioned difficulties. In these techniques the constraints are included as part of OF. The authors of reference [6] developed a method based on GA for optimal coordination. This method has miscoordination problem and does not include six current pairs. The detail description of the miscoordination problem is given in the next section. They also developed Evolutionary Algorithm [7], [8] and [9]. This method has the same problem as the previous. In Reference [10] a comparison between the linear programming method and genetic algorithm with similar OF of [6] has been made. The results have shown that the technique used in the paper is better than LP method.

The above mentioned GA techniques are improved by adding a new expression to the $\mathrm{OF}$ such that the miscoordination problems are solved for the fault just to be close to the C.B of the primary relay. Also the algorithm can handle both continues and discrete time setting multipliers (TSM) or time dial settings (TDS) [11]. The way of including the fuses in coordination process using GA is given. In other words a parameter which specified the type and size of fuses curves are taken into account [12].

In this paper, a new flexible and accurate method based on GA is presented in which the followings are included:

a) $\mathrm{OF}$ is improved by removing the redundant term to get simplicity and efficiency.

b) In the proposed OF of GA, six current pairs for each $\mathrm{P} / \mathrm{B}$ relay are considered. Therefore different network configuration, different fault locations and line outages have been taken into account.

c) Because of possessing high simplicity efficiency, flexibility, capability mentioned in (a) and (b) the method will be suitable for protection coordination of interconnected networks.

\section{GA APPLICATION TO RELAY CO-ORDINATION}

This section is devoted to review the notation and concept of GA application to overcurrent relays coordination presented in references [11], [12] to give a better understanding and coherency to this paper.

The flow diagram of the GA application to relay coordination is shown in Fig. 1.

The key variable in the GA is the chromosome and it consists of all relay TSM's or TDS's. The structure of the chromosome is shown in Fig. 2.

It should be noted that in the method for both discrete and continuous values of TSM's or TDS's solving the problem is made by considering that TSM's or TDS's are continuous initially. But, for discrete TSM's or TDS's (chromosomes), after the completion of each iteration and before starting the evaluation part of the procedure, the obtained TSM's or TDS's are rounded to the next allowable discrete value marked on the relays. That means the optimal OF for rounded TSM's or TDS's which solves inherently miscoordination is automatically selected. Of course for relays with continuous TSM or TDS, the inversion of TSM or TDS to discrete values is not made [11].

OF of reference [11], is written below

$$
\begin{gathered}
\mathrm{OF}=\alpha_{1} \sum_{i=1}^{N} t_{i}^{2}+\alpha_{2} \sum_{k=1}^{P}\left(\Delta t_{m b k}-\beta_{2}\left(\Delta t_{m b k}-\left|\Delta t_{m b k}\right|\right)\right)^{2} \\
\Delta t_{m b k}=t_{b k}-t_{m k}-C T I
\end{gathered}
$$

where

$\Delta t_{m b k}$ is the operation time difference with critical time interval for $k$ th relays pair, $t_{i}$ is $i$ th relay operating time for a fault close to the $\mathrm{CB}$ of the $i$ th relay, $t_{m k}$ and $t_{b k}$ are the operating times of the main and backup relays for a fault exactly close to the $\mathrm{CB}$ of the main relay, $N$ 
is the number of relays, $P$ is the number of $\mathrm{P} / \mathrm{B}$ current pairs, $k$ represents each $\mathrm{P} / \mathrm{B}$ current pair and varies from to $P$ and $i$ represents each relay and varies from to $N, C T I$ (Critical time interval) is the coordination time interval and can be taken to be 0.3 or $0.4 \mathrm{sec}$ depends on the accuracies of the relays curves, current transformers (CTs) overshoot and speed of the primary relay Circuit Breaker (CB).

In (1) $\beta_{2}$ is the parameter to consider the miscoordination, $\alpha_{1}$ and $\alpha_{2}$ are used to control the weightings of $\sum t_{i}^{2}$ and $\sum\left(\Delta t_{m b}-\beta_{2}\left(\Delta t_{m b}-\left|\Delta t_{m b}\right|\right)\right)^{2}$ respectively. The detail description of $\mathrm{OF}$ and its problems are given in section 3 .

The smaller the evaluated objective value, the better the chromosome. It should be noted that $\mathrm{OF}$ is composed only for one pair $(\mathrm{P} / \mathrm{B})$ currents. In other words fault location is considered to be close to the primary relays and no configuration changes were taken into account. The other stages of GA application to relay coordination are shown in Fig. 1 [11].

\section{PROBLEM STATEMENT}

The recent existing intelligent GA method can solve the miscoordination problem only for faults to be close to the $\mathrm{CB}$ of the primary relays (close in faults). Of course the relative currents in the primary and backup relays which are called one current pair have been considered. However, to cover fully coordination for different fault locations and line outages etc, two further developments are needed:

a) The modification of OF such that the GA method to be optimal, efficient and simple.

b) The modification of OF such that the method capable of solving the miscoordination of interconnected networks for the cases such as different fault locations, lines outages etc which is referred as to six current pairs.

The detailed description of the problems is given below.

To clarify the miscoordination problem of the existing method and make the OF of Section 2, (1) simple and more efficient, the equation is again taken into account. As can be seen (1) includes two expressions. The second expression is $\alpha_{2} \sum_{k=1}^{P}\left(\Delta t_{m b k}-\beta_{2}\left(\Delta t_{m b k}-\right.\right.$ $\left.\left.\left|\Delta t_{m b k}\right|\right)\right)^{2}$. To describe the role of the second expression, first consider $\Delta t_{m b k}$ to be positive. In this case, the mentioned expression will be equal to $\alpha_{2} \sum_{k=1}^{P}\left(\Delta t_{m b k}\right)^{2}$, however in the case $\Delta t_{m b k}$ is negative then $\left(\Delta t_{m b k}-\right.$ $\left.\left|\Delta t_{m b k}\right|\right)=2 \Delta t_{m b k}$, therefore the expression will be equal to $\alpha_{2} \sum_{k=1}^{P}\left(\left(1-2 \beta_{2}\right) \Delta t_{m b k}\right)^{2}$. Clearly, for positive values of $\beta_{2}\left(\beta_{2}>1\right)$, the value of (1) is large. This is because the value of $\beta_{2}$ is normally large according to Section 5 . Therefore based on a concept of the evaluation and selection parts of GA in Fig. 1, the values with less optimal OF values $i e$ (greater values) given in (1) are not selected. In other words, the TSM or TDS set that belongs to negative $\Delta t_{m b k}$ which shows the miscoordination is not chosen.

Although the method of reference [11] can solve miscoordination for close in faults, however by considering the situation in deep it is cleared that the OF can be improved in terms of simplicities, optimality and efficiency. In this regards, the second expression of (1) is analyzed again. By considering the first part of second expression ie first $\Delta t_{m b}$ of $\left(\Delta t_{m b k}-\beta_{2}\left(\Delta t_{m b k}-\left|\Delta t_{m b k}\right|\right)\right)$, it can be understood that this part can not help the optimization problem and it is redundant. Because for the first case (positive value of $\Delta t_{m b}$ ), this part makes OF greater and for the second case (negative value of $\Delta t_{m b k}$ ) makes it smaller. Whilst for optimization problem the mentioned $\Delta t_{m b k}$ should act vice versa. Of course because the value of $\beta_{2}$ is large, the effect of this $\Delta t_{m b k}$ in the first part of the second expression of case 2 is low but for case 1 is high.

Alternatively, when $\Delta t_{m b k}$ is positive, this part $\left(\sum\left(\Delta t_{m b k}\right)^{2}\right)$ is appeared as the whole second expression. Now solving the equation of OF with first expression having $\sum t_{i}^{2}$ and second expression having $\sum\left(\Delta t_{m b k}\right)^{2}$ for six current pairs which will be described in the next section make the problem to be sophisticated compared to the new OF of Section 4. As a results of above discussion, the existing $\mathrm{OF}$ needs to be modified for more simplicity and efficiency.

The existing GA method considers close in fault only ([11] and [12]). Line outages, high speed instantaneous elements, different fault locations on the line under consideration are not taken into account.

It has been described in [4] if the fault is considered to be close in fault and other fault places or line outages are not considered, miscoordinations may occur. To include all possibilities of fault locations and configuration changes which can occur in the real interconnected power systems, six current pairs are applied in some traditional coordination methods [4]. Six current pairs will be described in Section 4.

Therefore similar to ordinary coordination method, the mentioned six current pairs in genetic optimal coordination methods must be included. To include that, six related constraints must be introduced. The constraints belong to the close in fault, far end fault, the location of high speed instantaneous relays and different line outages.

\section{PROPOSED METHOD}

The flow diagram of the new method is the same as Fig. 1, however the difference between the new flowchart and Fig. 1 is the expression in the evaluation block. The novelty of the new method is in this block and six current pairs consideration which will be described below.

In this paper an OF of (1) is replaced by (3).

To make OF of reference [11] to be simple and efficient, the first part of the second expression of OF is removed. 
Table 1. Relay Characteristic Coefficients

\begin{tabular}{lccccc}
\hline Coefficient & $a_{1}$ & $a_{2}$ & $a_{3}$ & $a_{4}$ & $a_{5}$ \\
Value & 1.98772 & 8.57922 & -0.46129 & 0.0364465 & -0.000319901 \\
\hline
\end{tabular}

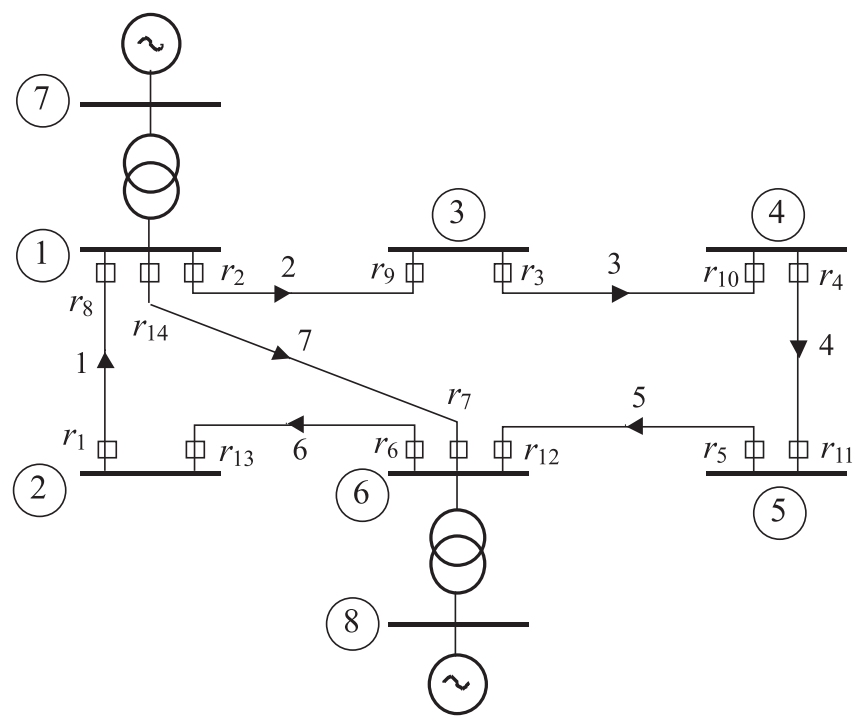

Fig. 3. Sample Network (8 Buses)

The justification of that has been given in Section 3 . Therefore the new equation is defined as

$$
\mathrm{OF}=\alpha_{1} \sum_{i=1}^{N} t_{i}^{2}+\beta_{3} \sum_{k=1}^{P}\left(\Delta t_{m b k}-\left|\Delta t_{m b k}\right|\right)^{2}
$$

where $N$ is number of relays, $P$ is number of $\mathrm{P} / \mathrm{B}$ current pairs ( ie number of relative $\mathrm{P} / \mathrm{B}$ currents for close in fault of each relay), represents each $\mathrm{P} / \mathrm{B}$ current pair and varies from to $P$ and $i$ represents each relay and varies from to $N$. $\beta_{3}$ is equal to $\alpha_{2} \beta_{2}^{2}$ because only the first part of the second expression has been removed and it is easy to compare of performance with (1).

It should be noted that the first term of the new $\mathrm{OF}$ can be squared or not. It remains as (1), because the (3) is modified previous one and enable us to compare with it. The second term is squared because for optimization technique the term can be either absolute values or squared. Otherwise the negative and positive values are added and do not meet the optimal coordination requirements. As a result the second term is also considered to be squared because the $\mathrm{OF}$ comparison with previous one can be made.

The important point in (3) is when the constraint is satisfied $\Delta t_{m b k}>0$, then the second part of equation becomes zero whilst the second term of existing (1) becomes $\alpha_{2} \sum_{k=1}^{P}\left(\Delta t_{m b k}\right)^{2}$, ie the new OF becomes smaller than existing OF. Therefore the chromosomes (TSM's) belong to this case using new $\mathrm{OF}$ are granted more opportunities than the existing method to survive. If the constraint is not satisfied ie $\Delta t_{m b k}<0$ and the second part (in the bracket) of the formula becomes $2 \Delta t_{m b k}$. In this case the second part $\beta_{3} \sum_{k=1}^{P}\left(2 \Delta t_{m b k}\right)^{2}$ is slightly greater than the relative expression of the previous method i.e. $\alpha_{2} \sum_{k=1}^{P}\left(\Delta t_{m b k}-2 \beta_{2} \Delta t_{m b k}\right)^{2}$ for $\alpha_{2}>1$ and $\beta_{2}>1$. Therefore compared to the existing method the chromosomes (TSM's) of this case (miscoordination) are granted less chance than the existing OF to survive. Obviously when $\Delta t_{m b k}>0$, the role of new OF is significant.

To cover co-ordination for six current pairs, for each $\mathrm{P} / \mathrm{B}$ relays the expression $\left(\Delta t_{m b k}-\left|\Delta t_{m b k}\right|\right)^{2}$ is written for six current pairs ie becomes $\sum_{k=1}^{P} \sum_{j=1}^{6}\left(\Delta t_{m b k}-\right.$ $\left.\left|\Delta t_{m b k}\right|\right)^{2}$, where $k$ represents each $\mathrm{P} / \mathrm{B}$ relays and $j$ represents each current pair.

Six current pairs $i e$ the relative currents of primary and backup relays are summarized below:

$j=1$ represents current pair No. $1(\mathrm{CP} \# 1)$ which means the fault is on the far end bus or line-end fault and lines outages are such that the current flowing through backup relay is maximum.

$j=2$ is for $\mathrm{CP} \# 2$. The fault location of $\mathrm{CP} \# 2$ is similar to $\mathrm{CP} \# 1$ but the current through the primary relay is minimum.

$j=3$ is represented by $\mathrm{CP} \# 3$ which consider close in fault but the lines outages are such that the current of backup relay is maximum.

$j=4$ is indicated by $\mathrm{CP} \# 4$ which consider the fault to be at a point such that the current of the primary relay to be equal to the highest instantaneous element current setting.

$j=5$ is shown by $\mathrm{CP} \# 5$, if the high set instantaneous element exists, the relevant current of each $\mathrm{P} / \mathrm{B}$ relay is the mean of current pairs $2 \& 4$. However if high set instantaneous element does not exist, then the mean of current pairs $2 \& 3$ is considered. 
Table 2. P/B Pair Data

\begin{tabular}{ccc}
\hline Row & Primary Relay & Backup Relay \\
\hline 1 & 8 & 9 \\
2 & 8 & 7 \\
3 & 2 & 7 \\
4 & 2 & 1 \\
5 & 3 & 2 \\
6 & 4 & 3 \\
7 & 5 & 4 \\
8 & 6 & 5 \\
9 & 6 & 14 \\
10 & 14 & 1 \\
11 & 14 & 9 \\
12 & 1 & 6 \\
13 & 9 & 10 \\
14 & 10 & 11 \\
15 & 11 & 12 \\
16 & 12 & 14 \\
17 & 12 & 13 \\
18 & 13 & 8 \\
19 & 7 & 5 \\
20 & 7 & 13 \\
\hline
\end{tabular}

Table 3. Current Setting Data

\begin{tabular}{ccc}
\hline Relay number & Load current & Pickup current \\
\hline 1 & 416 & 500 \\
2 & 666 & 800 \\
3 & 500 & 600 \\
4 & 666 & 800 \\
5 & 458 & 550 \\
6 & 458 & 550 \\
7 & 541 & 650 \\
8 & 458 & 550 \\
9 & 450 & 540 \\
10 & 458 & 550 \\
11 & 541 & 650 \\
12 & 458 & 550 \\
13 & 500 & 600 \\
14 & 666 & 800 \\
\hline
\end{tabular}

$j=6$ is for $\mathrm{CP} \# 6$, the fault point is the same as case $1 \& 2$ but the ratio of the backup relay current to the primary relay current is minimum.

It should be noted that for finding the relays operating times, a more common formula for approximating the relay characteristics is used [1]

$\frac{t}{\mathrm{TSM}}=a_{1}+\frac{a_{2}}{M-1}+\frac{a_{3}}{(M-1)^{2}}+\frac{a_{4}}{(M-1)^{3}}+\frac{a_{5}}{(M-1)^{4}}$

where, $M$ is is the ratio of relay current to the relay setting $\left(M=\frac{I_{s c}}{I_{b}}\right), I_{b}$ is relay setting and $I_{s c}$ is short circuit current, $a_{1}, a_{2}, a_{3}, a_{4}$ and $a_{5}$ are scalar quantities which characterize the particular device being simulated. $t$ is relay operating time.

Although today, digital relays with IEC and ANSI curves are used, however for existing static and electromechanical relays either IEC or ANSI formula or the equation (4) can be used. Equation (4) is more accurate for the static and electromechanical relays [13]. In future all the relays will be replaced by digital ones and therefore the simulating the inverse of $\mathrm{O} / \mathrm{C}$ relay will be vanished.

\section{TEST RESULTS}

\subsection{Sample 1}

\section{a) Network and Protection Data}

Figure 3 consists of 7 lines, 8 buses and 2 transformers. It is assumed that all the lines are protected by overcurrent relays and the overcurrent relays are normal inverse type and the relay characteristic is formulated by (4). Therefore on the sample network it is assumed the overcurrent relays are the main protection for the feeder under consideration and backup of them is the $\mathrm{O} / \mathrm{C}$ relays installed on the adjacent feeders of the main feeder. In other word the same protection system as references [6] and [10], has been taken into account. This is because the comparison results with OF of reference [6] and [10] to be meaningful. $a_{1}, a_{2}, a_{3}, a_{4}, a_{5}$ for the particular overcurrent device (normal inverse) is given in Tab. 1.

It is also assumed that TSM's of the relays continues and TSM's varies from 0 to 1 . The network information is given in reference [11]. In the references [10] and [14] also this network is used but the information are based on $150 \mathrm{MVA}$ and $150 \mathrm{KV}$, whilst in this paper, the same as reference [11], they are based on $100 \mathrm{MVA}$ and $150 \mathrm{KV}$. The information is not given here because of space limitation.

Table 4. Six Current Pairs for Relays No.8 \& 9

\begin{tabular}{ccc}
\hline Current Pair & Relay No.2 current & Relay No 1 current \\
\hline 1 & 2561.46 & 33.00 \\
2 & 2561.46 & 33.00 \\
3 & 3866.05 & 834.17 \\
4 & 3344.00 & 139.69 \\
5 & 2952.67 & 86.35 \\
6 & 2561.46 & 33.00 \\
\hline
\end{tabular}

Table 5. GA Parameters

\begin{tabular}{cc}
\hline GA parameters & value \\
\hline Number of generation & 2000 \\
Size of population & 100 \\
Initial population & random \\
Mutation & 1.0 \\
\hline
\end{tabular}

To obtain the OF, six pair short circuit (SC) currents of the backup and primary relays must be calculated on different locations of the lines of the primary relays. The relevant information including $\mathrm{P} / \mathrm{B}$ relays numbers and pickup current settings for the relays are given in Tables 2 
and 3. Also the information including SC currents related to six current pairs flowing in the primary and backup relays numbers $8 \& 9$ is given in Table 4 .

The control parameters of GA are given in Table 5 .

As described in the previous section, to compose OF, the determination of $\alpha_{1}, \beta_{2}, \alpha_{2}$ of (1) and $\beta_{3}$ of (3) is essential. For testing the effectiveness of GA for the purpose of overcurrent relays coordination, several trials with different values of $\alpha_{1}, \beta_{2}$ and $\alpha_{2}$ are tested in this paper. The variations of $\alpha_{1}, \beta_{2}, \alpha_{2}$ and $\beta_{3}=\alpha_{2} \beta_{2}^{2}$ values are listed in Table 6 . It should be noted that the value of $\beta_{3}$ is selected to be coherent with the description given in Section 4. Any value of $\beta_{3}$ greater than 100 does not change the results.

Table 6. Parameter Variations

\begin{tabular}{cccccc}
\hline $\begin{array}{c}\text { Case } \\
\text { number }\end{array}$ & $\alpha_{1}$ & $\beta_{2}$ & $\beta_{3}$ & $\alpha_{2}$ & Co-ordination method \\
\hline Case 1 & 1 & - & 20000 & - & 6 pair, final OF \\
Case 2 & 1 & - & 2 & - & 6 pair, final OF \\
Case 3 & 1 & 100 & - & 2 & 6 pair, previous OF \\
Case 4 & 1 & 100 & - & - & 1 pair, final OF \\
Case 5 & 1 & 100 & - & 2 & 1 pair, previous OF \\
Case 6 & 1 & 0 & - & 100 & 6 pair, traditional old OF \\
\hline
\end{tabular}

\section{b) Results and Discussion}

By applying the GA with selected values to the network of Fig. 3, the output results are obtained. In the cases 1 and 2 , six current pairs by considering the new equation of $\mathrm{OF}$ are taken into account. Case 3 is related to 6 current pairs using previous OF. However cases 4 and 5 consider only close in fault for the new and the previous methods respectively. Case 6 uses OF described in reference [6] for 6 pairs which is referred to traditional old OF. Table 7 contains 3 parts. Part a includes TSM's of different relays. In the second part $b$, the operating times of the relays for close up fault or fault on the location where high set instantaneous current setting flow through the relay if high set instantaneous elements (H$\mathrm{S})$ exist, are given. Table 7, part c includes the difference between discrimination times for $\mathrm{P} / \mathrm{B}$ relays and CTI. As mentioned in Section 2, part c, CTI can be selected to be 0.3 or 0.4 . For relays and CTs with high accuracies and C.B fast operating times, 0.3 is used. In this example $0.3 \mathrm{sec}$ is chosen.

There are $20 \mathrm{P} / \mathrm{B}$ set relays for Figure 3, therefore for each current pair twenty $\Delta t$ exist. For six current pairs, the $\Delta t$ rows will be $20 * 6=120$. As a result, the whole table seems too long. Some backup relays currents are less than 1.6 times than the line current settings of the relevant relays, they are not included in Table 7. Example of that is coordination between relay 8 and 9 . By considering the first row of Table 4 the primary SC current is $2561.46 \mathrm{~A}$ whilst the backup relay current is only $33 \mathrm{~A}$. This is because relay 8 which is the primary relay is connected to a generator-transformer bus. In such a case there is no need to study coordination. Other primary relays with similar situation are also connected to generator-transformer buses. In the following discussion, it will be seen that some selected pairs are only included in part c of Table 7. This is because, for the other current pairs, the backup relay currents are less than 1.6 times the current settings.

Six relevant columns of Table 7 represent different methods application results. The parameter variations for six cases are given in Table 6 . As an example if the value of $\beta_{2}$ is chosen to be about 100 times $\alpha_{1}$, they will be the suitable parameters, Therefore $\alpha_{1}=1, \beta_{2}=100$ are chosen. When $\alpha_{1}=1, \beta_{2}=1$ and therefore $\beta_{3}=2$, the role of miscoordination is not active. So this case (case 2) is also selected for comparison. Of course in all cases the values greater than -0.05 and less than zero are considered as zero. This means in equation (2), when $-0.05 \leq \Delta t \leq 0$ the variation compared to $0.3 \mathrm{sec}$ is very small and can be neglected and therefore $t_{b}-t_{m} \approx 0.3 \mathrm{sec}$. In case 1 which is represented in the column 1 of the result, all $\Delta t$ values are either zero or positive. In other words in column 1, 6 negative numbers are seen, however all of them are greater than -0.05 and less than zero. Therefore they are considered to be zero. This means there is no miscoordination. Therefore this is the best solution.

In case 2 (column 2) although the operating times of the relays are less than the previous case, 11 miscoordinations are seen. They are marked in Table 7 . In case 3, the recently published $\mathrm{OF}$ is used. The relays operating times plus discrimination times between $\mathrm{P} / \mathrm{B}$ relays are too longer. By considering the forth column (case 3 ) of part (b) of Table 7 it can be seen among 14 relays, 8 of them ie relays $2-5$ and 9-12 have much longer operating times and the remaining 6 are almost the same compared to case 1 . Although both cases 1 and 3 have no miscoordination, but relays operating times of case 3 are longer, therefore case 1 which posses two advantages compare to case 3 with one advantage is preferred. As a result the dependencies of TSM's to OF are complicated and the role of optimization of GA is decreased.

Case 4 shows the results of GA application to the network for 1 current pairs. Here, also 3 miscoordinations were found. Of course this case is obviously worse than case 1 . Case 5 which is the recently published OF and 1 pair, the operating times are greater than case 1 , and 3 miscoordination exist only for one current pairs. Also the operating times of the most relays for close up fault are either equal or greater than case 4 and consequently case 1.

In Case 6 which is the traditional old GA case, reference [6], 10 miscoordinations were found.

Therefore Case 1, is consequently selected as a suitable case. In this case miscoordination does not exist, the relay operating times are rather low. Therefore it is revealed that the new OF is more optimal. 
Table 7. GA Output

(a)

(c)

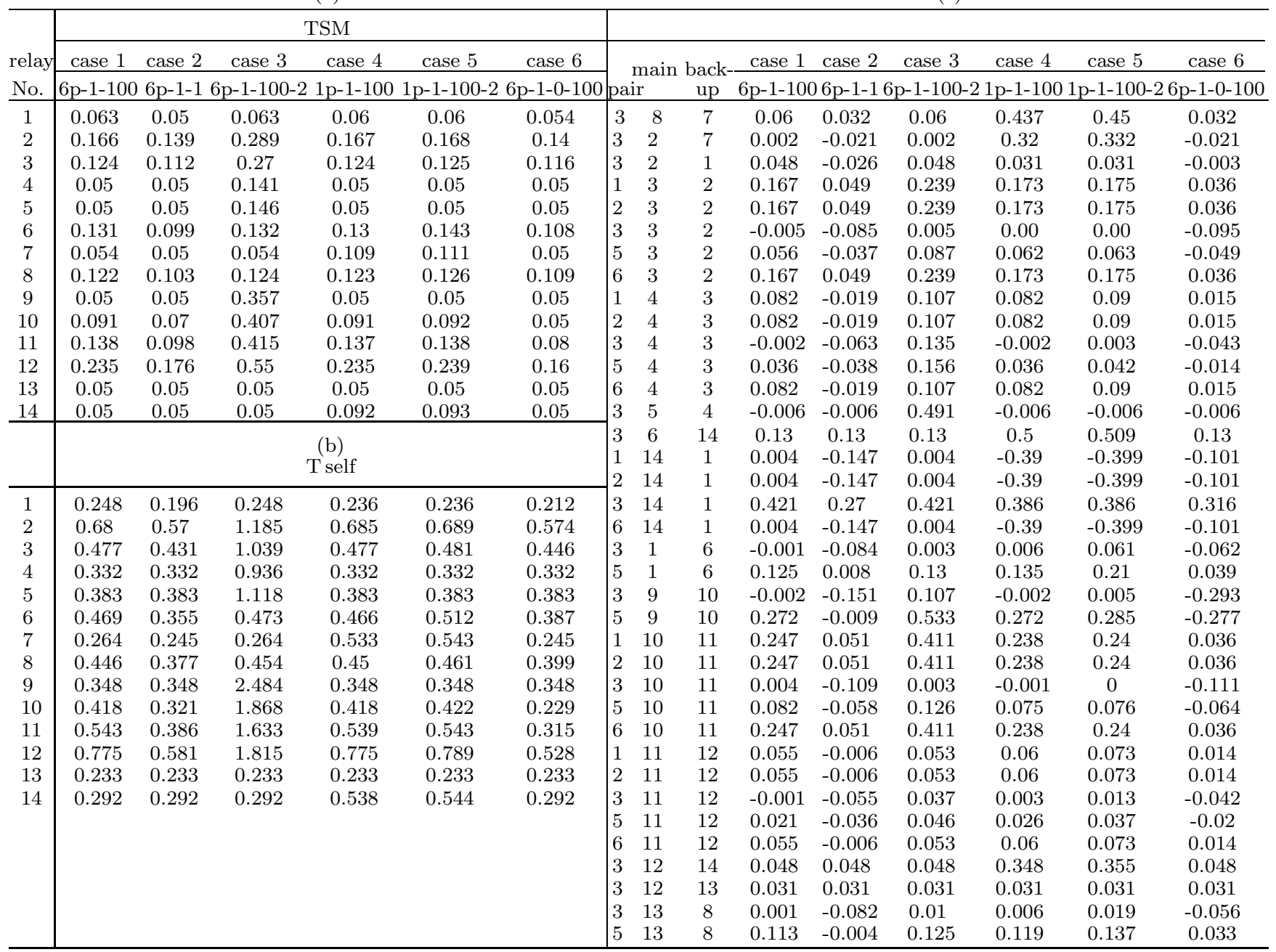

Table 8. Discrimination Times, Average TSMs and OTs Summary for IEEE 30 Buses Relays

\begin{tabular}{|c|c|c|c|c|c|c|}
\hline \multirow{2}{*}{ Cas } & Cas & 2 & Cas & Case 4 & Case 5 & \\
\hline & $6 \mathrm{p}-1-$ & $6 \mathrm{p}-1-1$ & $0-1-100-2$ & $1 p-1-100$ & $1 p-1-100-2$ & $6 \mathrm{p}-1$ \\
\hline & 246 & 0.2 & 2470.746 & 195 & $186 \mathrm{C}$ & 1 \\
\hline$\Delta$ & -0.068 & -14.11 & 0 & -12.16 & -13.25 & 73 \\
\hline & 0.121 & & 0.194 & & & \\
\hline OT & 0.541 & 0.258 & 0.885 & 0.367 & 0.385 & 0.652 \\
\hline
\end{tabular}

\subsection{Sample 2}

\section{Information Data}

The next example is IEEE 30 buses system which can be considered as a meshed subtransmission / distribution system. The network consists of 30 buses (132 and $33 \mathrm{kV}$ buses), 37 lines, 6 generators, 4 transformers and 86 OC relays. The same OC protective relays as 5.1 section are assumed for the network shown in Fig. 4. The generators, transmission lines and transformers information are given in reference [15].
Results and Discussion

Again GA with selected values of $\alpha_{1}, \beta_{2}, \alpha_{2}$ and $\beta_{3}$ given in Table 6 has been applied to the network of Fig. 4. The summary of discrimination times, the average of TSMs and the relays operating times (OTs) are shown in Table 8.

The four rows of Table 8 consist of $\Delta t$ greater than zero (fully coordination), $\Delta t<0$ (misscoordination), the average of TSMs of all relays and average of relays operating times for faults close to the relays respectively. The information of each row is for 6 defined cases. The 
(G) Generators

(C) Synchronous condensors
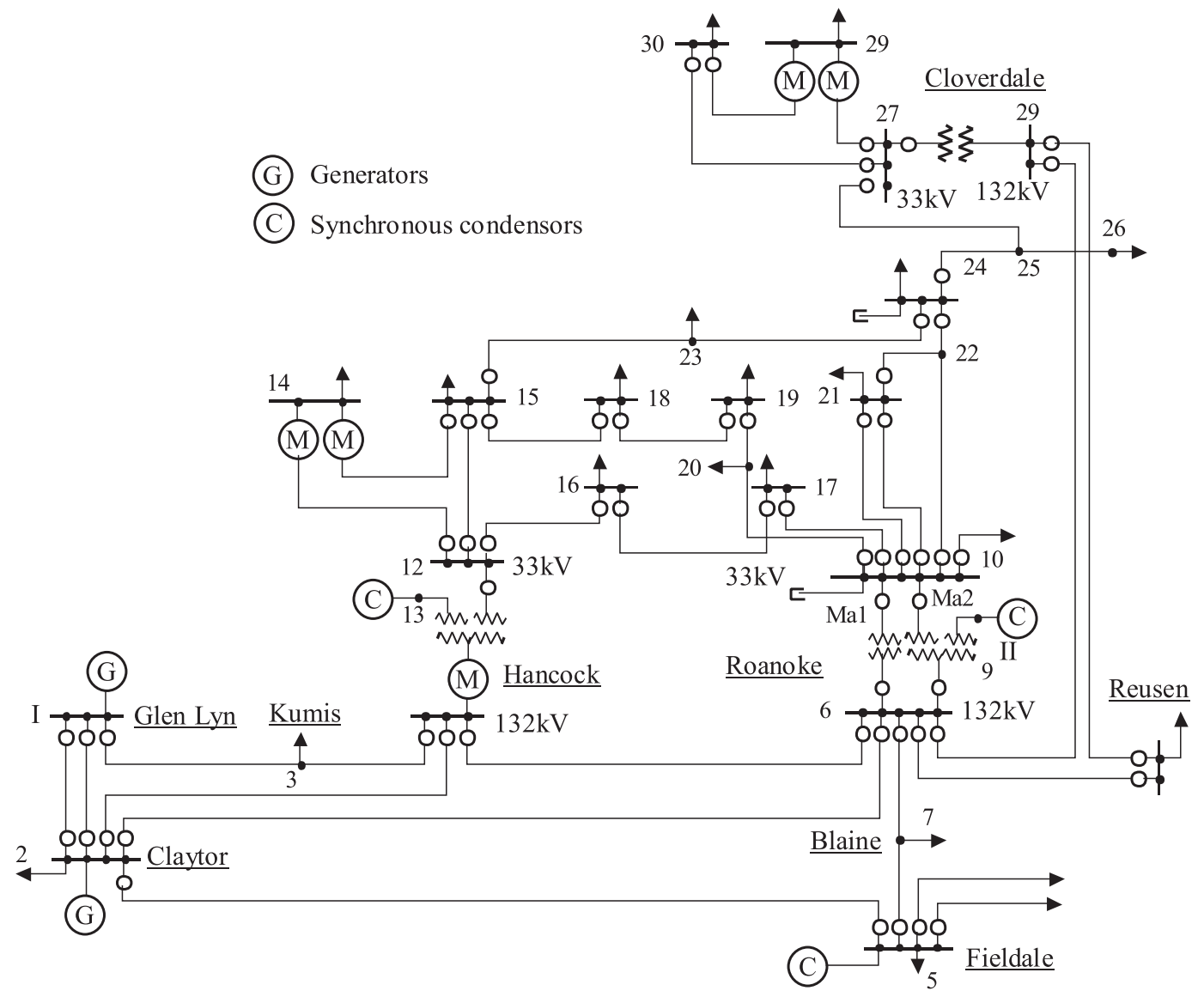

Fig. 4. IEEE 30 Buses Network

information values related to first row for each case are number of $\mathrm{P} / \mathrm{B}$ relays and average of $\Delta t>0$. However the values of second row are number of $\mathrm{P} / \mathrm{B}$ relays with $\Delta t>0$ and the sum of the related relays pairs $\Delta$. This is because for $\Delta t>0$, the average amount and for the second row the sum can more help the method evaluation. It should be noted that again $-0.05 \leq \Delta t \leq 0$ is considered to be zero.

The values in the Table 8 are obtained from the tables of TSM and operating times of all the relays and $\Delta t$ of $\mathrm{P} / \mathrm{B}$ relays. The tables are similar to Table 7 , parts $\mathrm{a}, \mathrm{b}$ and c. Because of space limitation and long tables they are not given in this paper.

Case 1 to Case 6 parameters and situation are exactly the same as example of Section 5.1. The fault position in Table 8 is either close up fault or the location where the high set instantaneous current flow through the relay if high set instantaneous element $(\mathrm{H}-\mathrm{S})$ exist. Here also there are $228 \mathrm{P} / \mathrm{B}$ set relays for Figure 4. Again similar to example 1 , for this network also it will be shown even that case (1) is the suitable one.

For case 1 the average operating times is 0.541 , while the relative average operating times of case 2 is 0.258 . Although the average operating times is lower in case 2, however 86 miscordinations have been arised. Both cases have used the new OF, but different parameters of OF have caused the different results. Consequently because of many miscoordinations in casetwo, case 1 is preferred.
Similar to example of Section 5.1, in case 3 there is no miscoordination, but the average relay operating times of case 3 is 0.885 which is much longer than case 1 and the average discrimination times of $\mathrm{P} / \mathrm{B}$ relays is also greater than case 1 . That means for primary relays or primary C.B failures, longer tripping times of the backup relays would take. Therefore in spite of having 1 small miscoordination in case 1 , because of lower mentioned operating and discrimination times, this case is selected as suitable one. Cases 4 and 5 which are for 1 current pairs using the new and previous OF, 52 and 61 miscoordinations have been resulted respectively. It should be noted here for cases 4 and 5 the coordination has been done with one current pairs only, whilst the assessment of miscoordination has been made for 6 current pairs. As all current pairs have not been considered during the coordination processes of the cases, naturally miscoordinations are arisen. The assessment is made to show the advantage of coordination with 6 current pairs compared to the coordination with one pair.

Finally for case 6 which is again the traditional old GA ([6], [8] and [10]), 73 miscoordinations exist.

In this section (Section 5) the results of the three methods application ie the new one with two other methods used in references [7] and [11] are compared. Two different networks with different configurations ( $i e$ IEEE 30 buses \& 8 buses system have been selected as the test systems). 
From the results it has been shown that the new method is better than the other existing ones.

\section{CONCLUSION}

A new overcurrent relay coordination method based on the Genetic algorithm (GA) has been developed. In the proposed method, a new objective function $(\mathrm{OF})$ is introduced which includes six current pairs of $\mathrm{P} / \mathrm{B}$ relays and the redundant term has been removed. Therefore objective function is more optimal than the previous one, and no miscoordination exists for different fault locations and under different line outages conditions. The computer program has been tested on two different network configurations. From the obtained results, it has been shown that the new approach compared to the previous techniques is more optimal, flexible, efficient and successful.

\section{REFERENCES}

[1] ABYANEH, H. A.-MAJIDAl-DABBAGH-KAREGAR, H. K.-SADEGHI, S. H. H.-KHAN, R. A. J.: A New Optimal Approach for Coordination of Overcurrent Relays in Interconnected Power Systems, IEEE Transaction on Power Delivery 18 No. 2 (April 2003).

[2] KARGAR, H. K.-ABYANEH, H. A.-OHIS, V.-MESHKIN, M. : Pre-Processing of the Optimal Coordination of Overcurrent Relays, Electric Power Systems Research, Elsevier 75 No. 2-3 (June 2005).

[3] BIRLA, D.-MAheshWARI, R. P.-GUPTA, H. O.: TimeOvercurrent Relay Coordination: A Review, International Journal of Emerging Electric Power Systems 2 No. 2 (2005).

[4] Westinghouse Electric Corporation, Relay Instrument Division Coral Springs: Applied Protection Relaying, Florida 33065, copyright 1982, chapter 10.

[5] ABYANEH, H. A.-KEYHANI, R. : Optimal Co-Ordination of Overcurrent Relays in Power System by Dual Simplex Method, in: Proc. 1995 AUPEC Conf., vol. 3, Perth, Australia yr1995, pp. 440-445.

[6] SO, C. W.-LI, K. K.-LAI, K. T.-FUNG, K. Y. : Application of Genetic Algorithm for Overcurrent Relay Coordination, Proc. IEE Conf. Developments in Power System Protection, 1997, pp. 66-69.

[7] SO, C. W.-LI, K. K.: Overcurrent Relay Coordination by Evolutionary Programming, Electric Power Systems Research 53 (2000), 83-90.

[8] SO, C. W.-LI, K. K.: Time Coordination Method for Power System Protection by Evolutionary Algorithm, IEEE Transactions on Industry Applications 36 No. 5 (Sep-Oct 2000), $1235-1240$.

[9] SO, C. W.-LI, K. K.: Intelligent Method for Protection Coordination, IEEE International Conference Of Electric Utility Deregulation Restructuring and Power Technology, Hong Kong, Apr 2004.

[10] ZEINELDIN, H.-El-SAADANY, E.-SALAMA, M. : Optimal Coordination of Directional Overcurrent Relay Coordination, IEEE Power Engineering Society General Meeting, San Francisco, June 2005.

[11] RAZAVI, F.-ABYANEH, H. A.-Al-DABBAGH, M.-MOHAMMADI, R.-TORKAMAN, H.: A New Comprehensive Genetic Algorithm Method for Optimal Overcurrent Relays Coordination, Electric Power Systems Research 78 No. 4 (Apr 2008), 713-720, Elsevier.
[12] ASKARIAN, H.-RAZAVI, M., F.-KHODDAMI, M.-TORKAMAN, H. : A New Genetic Algorithm Method for Overcurrent Relays and Fuses Coordination, IEEE Powertech, Switzerland, July 2007.

[13] SACHDEV, M. S.-SINGH, J.-FLEMING, R. J. : Mathematical Models Representing TimeCurrent Characteristics of Overcurrent Relays for Computer Application, IEEE Transactions on Power Apparatus and Systems 131 (Jan 1978).

14] ZEINELDIN, H.-El-SAADANY, E.-SALAMA, M. A. : Optimal Coordination of Overcurrent Relays Using a Modified Particle Swarm Optimization, Electric Power Systems Research 76 No. 11 (July 2006), 988-995.

[15] Power system test cases, www.ee.washington.edu/resesrch/pstca.

Received 25 May 2009

Reza Mohammadi was born in Tabriz, Iran, on September 22, 1981. He received the BS and MS degree in electrical engineering from Iran University of Science and Technology (IUST) and Amirkabir University of Technology (AUT) in 2004 and 2007 respectively. Currently, he is $\mathrm{PhD}$ student in power engineering at AUT. His field of interest includes power system protection, distributed generation and intelligent methods.

Hossein Askarian Abyaneh was born in Abyaneh, Isfahan on March 20, 1953. He received the BS and MS degree both in Iran in 1976 and 1982 respectively. He also received another MS degree and PhD from UMIST, Manchester, UK in 1985 and 1988 respectively, all in electrical power engineering. He published over 100 scientific papers in international journals and conferences. Currently, he is a Professor with the Department of Electrical Engineering, AUT, Iran, working in the area of the relay protection and power quality.

Farzad Razavi received the BS, MS and PhD degrees from the AUT, Iran, in 1998, 2000 and 2007 respectively, all in power engineering. His employment experience included R\&D Counselor and R\&D Manager in Pars Tableau Company, Project Manager in Sepehr Company. His fields of interest included power system protection, mathematic and FACTS.

Majid Al-Dabbagh (M'90-SM'97) received the BEng and Master of Technical Science in Engineering degrees from Moscow Power Engineering University, Moscow, Russia, in 1968, and the MSc and PhD degrees from the University of Manchester Institute of Science and Technology, Manchester, UK, in 1973 and 1975, respectively. He was a Professor and Head of the Department of Electrical and Communication Engineering, Papua New Guinea University of Technology, Lae, Papua New Guinea. Between 1983 and 2006, he was with Royal Melbourne Institute of Technology, Melbourne, Australia. He is now with Hydro Tasmania Consulting, Melbourne Office, Melbourne.

Seyed H. H. Sadeghi (M'92) received the BS degree in electrical engineering in 1980 from Sharif University of Technology, Tehran, Iran, the MS degree from the University of Manchester Institute of Science and Technology, Manchester, UK, in 1984, and the PhD degree from the University of Essex, Essex, UK Between 1980 and 1983, he was appointed as a Research Assistant Professor at Vanderbilt University, Nashville, TN. From 1996 to 1997, he was a Visiting Professor with the University of Wisconsin, Milwaukee. He is presently a Professor of electrical engineering with Amirkabir University of Technology, Tehran, Iran. 